\title{
BMJ Open Integrated approach of nutritional and molecular epidemiology, mineralogical and chemical pollutant characterisation: the protocol of a cross-sectional study in women
}

\author{
Martina Barchitta, ${ }^{1,2}$ Annalisa Quattrocchi, ${ }^{1}$ Andrea Maugeri, ${ }^{1}$ Germana Barone, ${ }^{3}$ \\ Paolo Mazzoleni, ${ }^{3}$ Alfio Catalfo, ${ }^{4}$ Guido De Guidi, ${ }^{4,5}$ Maria lemmolo, ${ }^{6}$ \\ Nunzio Crimi, ${ }^{6}$ Antonella Agodi ${ }^{1,2}$
}

To cite: Barchitta M, Quattrocchi A, Maugeri A, et al. Integrated approach of nutritional and molecular epidemiology, mineralogical and chemical pollutant characterisation: the protocol of a cross-sectional study in women. BMJ Open 2017;7:e014756.

doi:10.1136/bmjopen-2016014756

- Prepublication history for this paper is available online. To view these files please visit the journal online (http://dx.doi.org/10.1136/ bmjopen-2016-014756).

Received 17 October 2016 Revised 14 December 2016 Accepted 17 February 2017

CrossMark

For numbered affiliations see end of article.

Correspondence to Professor Antonella Agodi; agodia@unict.it

\section{ABSTRACT}

Introduction: Environmentally-related health and disease are the result of the exposome, the totality of a person's environmental exposures, from all sources and routes, across their lifespan. Epigenetic phenomena, including DNA methylation, can be potentially modified by environmental and lifestyle factors, and result in environmental reprogramming of the genome for exposed individuals and for future generations of offspring.

Objective: The objective of the project is to evaluate the risk of DNA hypomethylation due to air pollution, Mediterranean diet adherence, folate intake, and demographic and socioeconomic factors, in healthy women living in the metropolitan area of Catania, Italy.

Methods and analysis: Non-pregnant healthy women will be enrolled in a cross-sectional study. Sociodemographic, lifestyle and dietary intake information will be collected. LINE-1 methylation will be measured by pyrosequencing. The participants' home addresses will be geocoded and each woman will be assigned to the closest monitoring station for particulate matter (PM) exposure assessment. Mineralogical-chemical characterisation of PM and cellular model assays will be performed. An integrated approach will be designed to estimate the combined possible effect of air pollution, Mediterranean diet adherence, folate intake and other lifestyle characteristics on LINE-1 methylation levels.

Ethics and dissemination: The project has been approved by the ethics committees of the involved institution and funded by the University of Catania (Finanziamento della Ricerca, FIR 2014). All participants will be fully informed of the purpose and procedures of the study, and signed written consents will be obtained. All the data collected will be treated confidentially and analysed in an aggregate and anonymous way. The results will be published in peerreviewed journals and communicated to local public health agencies, in order to provide essential information for timely and effective public health action.

\section{Strengths and limitations of this study}

- The present project integrates different individual exposures to investigate the associated epigenetic effects.

- The project design incorporates environmental and nutritional exposures in order to evaluate the associated epigenetic signatures of an individual's cumulative exposure.

- The relationship between dietary patterns and DNA methylation may be confounded by genetic factors that may result in LINE-1 hypomethylation through an interaction with folate status.

- The assessment of folate intake through a Food Frequency Questionnaire does not preclude measurement errors and may suffer from inaccuracies of volunteers' recall.

\section{INTRODUCTION}

The Mediterranean diet (MD) has been proposed as a healthy diet for disease prevention and health promotion. ${ }^{1}$ Moreover, the National Institute of Environmental Health Sciences (NIEHS) recommended that future environmental health research, and pollutant risk assessment approaches, incorporate nutrition and dietary practices, since nutritional factors may either increase the response to chemicals or reduce it. This approach recognises that environmentally related health and disease are the result of the totality of a person's environmental exposures, from all sources and routes, across the lifespan-the 'exposome'. The concept highlights the need for a more complete environmental exposure assessment in epidemiological studies. The exposome, therefore, complements the genome by providing a comprehensive description of lifelong exposure history. ${ }^{2}$ 
Epigenetics investigates heritable changes in gene expression that occur without changes in DNA sequence. ${ }^{3}$ Epigenetic modifications encompass a range of mechanisms including DNA methylation, histone modifications, histone variants and chromatin remodelling. The cellular epigenome is dynamic in response to environmental signals, modifiable during normal cell differentiation and is heritable in daughter cells. ${ }^{4}$ DNA methylation can be potentially modified by environmental and lifestyle factors, and results in environmental reprogramming of the genome for exposed individuals and for future generations of offspring. ${ }^{5}$ Long Interspersed Nucleotide Elements 1 (LINE-1), which are the most common repetitive elements of interspersed DNA repeats, and the most greatly methylated, have been proposed as a surrogate marker for estimating global DNA methylation level, ${ }^{6}$ even though growing evidence indicates that they could have specific and distinct cellular roles. ${ }^{7}$ Although LINE-1 methylation is not universally accepted as a marker of global methylation, ${ }^{8}$ it has been well established as having a biological role regulated by specific mechanisms and exposures. ${ }^{9}$ Hypomethylation of repetitive elements favours their activity as retrotransposable sequences and has been suggested to have deleterious effects on cells, initially through insertional mutations, ${ }^{10}$ and later by introducing genome instability through deletions and genomic rearrangements. ${ }^{7}$ The analysis of global DNA methylation levels, using LINE-1 as a biomarker, is one of the main aims of many researchers. Indeed, LINE-1 hypomethylation was observed in several types of cancer and was associated with a poor prognosis. ${ }^{11}$ Recently, two meta-analyses confirmed that LINE-1 methylation levels are significantly lower in cancer patients than in control samples, especially in certain cancer types. ${ }^{12} 13$

Folate is a critical methyl donor for several molecular pathways and its deficiency status has been associated with several adverse health conditions, ${ }^{14}$ including cancer. ${ }^{15}$ Folic acid plays an important role in the maintenance of genomic stability and different biomarkers of genomic instability have been proposed as being associated with folate status. Among those, the mitochondrial DNA (mtDNA) 4977-bp deletion, detected in some sporadic diseases and during aging, has been used as an mtDNA damage biomarker. In an experimental study in rodents, accumulation of mtDNA deletions in lymphocytes has been reported after folate deprivation. ${ }^{16}$ In humans, an increased frequency of mtDNA 4977-bp deletion has been associated with hepatocellular carcinoma risk and a high frequency of deletions was associated with low levels of folate. ${ }^{17}$ However, a recent study conducted in young healthy women reported that mtDNA 4977-bp deletions are maintained at low levels in lymphocytes despite the wide range of variation of folate intakes and folate status. ${ }^{18}$ Conversely, low fruit intake and folate deficiency were shown to be independent risk factors associated with low LINE-1 methylation levels. $^{19}$ In women with high grade cervical intraepithelial neoplasia (CIN), LINE-1 hypomethylation was shown to be influenced by methyl donor micronutrient intake, including folate, ${ }^{20}$ and thus a healthy diet may reduce the risk of CIN through higher LINE-1 methylation levels. $^{15}$

Because of the role of the methylenetetrahydrofolate reductase (MTHFR) gene in one carbon metabolism, polymorphisms, which alter folic acid metabolism, may affect DNA methylation profiles. ${ }^{21-23}$ Accordingly, several studies have hypothesised the relationship between two of these polymorphisms (MTHFR C677T and A1298C) and genomic DNA hypomethylation. ${ }^{24} 25$ However, to date, evidence is inconsistent as reported by Wang $e t a t^{26}$ in a recent meta-analysis.

Particulate matter (PM) exposure may induce carcinogenesis-related biological processes that may alter gene expression via DNA methylation mechanisms as well as in candidate proinflammatory genes. ${ }^{27} 28$ Numerous studies focused on Mediterranean sites considering the enrichment of aerosol concentrations in urban, suburban and rural zones. ${ }^{29}$ Other studies investigated the variability of regional background PM levels. ${ }^{30}$ However, the peculiar geological and environmental features make the metropolitan area of Catania particularly interesting since the explosive activity of Mount Etna frequently produces large volumes of gases and tephra.

Previous epidemiological studies conducted in women living in the metropolitan area of Catania showed high a prevalence of overweight/obesity and folate deficiency, ${ }^{18} 31$ a low level of red blood cell folate, high homocysteine concentrations and poor adherence to the MD. ${ }^{32} 33$ In addition, preliminary data suggested a variable distribution of global DNA methylation profiles in a cancer-free population. In particular, low consumption of fruit, a main source of folate, antioxidants and polyphenols, was associated with LINE-1 hypomethylation; thus, a dietary pattern characterised by a high fruit intake may protect against epigenetic modifications. ${ }^{19}$

The aim of the present project is to evaluate whether adherence to the MD, the intake of specific nutrients and the exposure to air pollutants are associated with LINE-1 methylation levels in healthy women in Catania, Italy. Specific objectives are: (1) to evaluate the association between diet-in terms of adherence to MD and of intake of folate and other micronutrients and LINE-1 methylation levels; (2) to analyse seasonal meteorological changes and chemical and mineralogical characteristics of PM; (3) to characterise polycyclic aromatic hydrocarbons (PAHs) and their derivatives; (4) to perform experiments on cellular systems exposed to PM of different origin to study the potential pro-oxidant, pro-inflammation, pro-fibrotic and DNA methylation effects; (5) to classify enrolled women based on air pollutant exposure data; (6) to design a model for estimating the combined effect of air pollution, MD adherence, folate intake and other lifestyle characteristics, including demographic and socioeconomic factors, physical activity and body mass index (BMI), on LINE methylation 
levels in healthy women living in the metropolitan area of Catania.

\section{METHODS AND ANALYSIS}

\section{Study design and structured questionnaire}

The project entitled 'Integrated approach of nutritional and molecular epidemiology, mineralogical and chemical pollutants characterisation and in vitro studies: Mediterranean diet and particulate matter exposure in women of the Catania metropolitan area' will run for 2 years. Non-pregnant healthy women will be enrolled in a cross-sectional study. The inclusion criteria are: (1) females aged between 13 and 50 years; (2) being nonpregnant; and (3) no current or previous self-reported history of severe diseases including cancer. A structured questionnaire has been designed in order to obtain information on sociodemographic and lifestyle data including smoking and physical activity. Level of education level will be recorded, and women will be classified into two categories: low (primary school, ie, $\leq 8$ years of school) and high (high school education or greater, ie, $>8$ years of school) education level. Employment status and job type will also be recorded to take into account occupational exposure. BMI will be calculated as weight $(\mathrm{kg})$ divided by height $\left(\mathrm{m}^{2}\right)$, based on criteria from the WHO. ${ }^{34}$

\section{Dietary assessment}

Dietary intake will be estimated by a validated semiquantitative Food Frequency Questionnaire (FFQ), as previously described. ${ }^{33}$ For each of the food items, women will be asked to report the frequency of consumption and portion size, using an indicative photo atlas, to estimate the amount of each food item and to minimise inaccuracies. Adherence to the MD will be assessed using the Mediterranean Diet Score (MDS), ${ }^{1}$ and women will be classified into three groups: MDS $\leq 25$ th percentile, defined as low adherence to the MD; MDS $>25$ th percentile but $\leq 90$ th percentile, defined as medium adherence to the MD; and MDS $>90$ th percentile, defined as high adherence to the MD. Folate and caloric intakes will be calculated using the USDA Nutrient Database (http://ndb.nal.usda.gov/), adapted to the Italian food consumption. Prevalence of folate deficiency will be estimated by comparing overall folate intake with the estimated average requirements (EAR), ${ }^{35}$ and intake of folic acid from supplements will be addressed. ${ }^{31}$

\section{Air pollution exposure assessment}

The participants' home addresses will be geocoded to assess air pollution exposure. In particular, georeferentiation using a Geographic Information System (GIS) of the home address of each woman will be performed and each woman will be assigned to the closest monitoring station.

The metropolitan area of Catania shows several weather control stations that monitor different measurements, including PM10 (but not lower sized particles), carbon monoxide, nitrogen dioxide, ozone and benzene. Data regarding the main air pollutants, for which routine measurements are performed by local authorities, will be retrieved.

PM2.5 measurements will be obtained by means of the mobile monitoring station. Accordingly, for PM2.5 qualitative and quantitative characterisation, different city districts, as well as different districts within $30 \mathrm{~km}$ of the city, will be included.

Thus, the place of residence will be crossed with data on PM levels detected in different areas of the city, in different seasons, and with data from the mineralogical and chemical characterisation. Finally, each woman will be classified on the basis of the different levels and types of exposure identified.

\section{Sample collection}

A high-volume impactor ECHO HiVol (TCR Tecora, Italy) will be used to obtain PM samples. In different sampling sites, samples of PM2.5 $(>2.5 \mu \mathrm{m})$ at $12 \mathrm{~m}^{3} /$ hour will be collected. $100 \mathrm{~mm}$ diameter glass fibre (AA99-016-9951CR) and quartz fibre (AA99-016-9950CR) filters will be used. The samplers will be set up close to the urban area, directly exposed to the exhaust emissions, and it will be located about $50 \mathrm{~m}$ from the road. The sampling will go on for 24 or 48 hours, at a flow rate of approximately $0.2 \mathrm{~m}^{3} / \mathrm{min}$. The impactor will be approximately calibrated every week. The total volume of the air sampled after 24 hours will be $288 \mathrm{~m}^{3}$. The size range is based on aerodynamic diameter. Aerodynamic size, rather than geometric size, determines the trajectory of a particle in a gas stream because it accounts for all three major aerodynamic factors: size, shape and mass density. The direct measurement of the impactors is 'equivalent aerodynamic diameter,' defined as the size of a spherical particle of mass density $1 \mathrm{~g} / \mathrm{mL}$ which has the same terminal settling velocity as the sampled particle. In contrast, microscope and light scattering methods of particle sizing do not account for mass density and aerodynamic size. ${ }^{36}$ Aerodynamic size is the most important parameter in particle work because it determines the penetration of particles in the human lung, the particle collection efficiency in pollution control equipment, and the transport and diffusion of particles in the ambient air. ${ }^{37}$ The filters will be weighed before and after sampling, after conditioning and drying in the dark. Finally, each filter will be covered with aluminium foil and then refrigerated $\left(-20^{\circ} \mathrm{C}\right)$ until use. $^{36}$

\section{Mineralogical-chemical characterisation of PM}

The mineralogical and chemical composition of sampled PM will be performed to determine the major sources contributing to the PM abundance. ${ }^{38}$ This issue assumes a key role due to the particular geographical, geological, meteorological and anthropogenic conditions of Catania affecting the presence of particular matter formed in different ways: (1) mineral dust-volcanic, soil and desert particles; (2) sea salt—halides and 
sulfates; (3) anthropogenic particles-carbonaceous particles.

The investigation strategy proposed consists of the use of different methodologies. X-ray diffraction on filters will obtain the bulk mineralogical composition of the PM sampled, while micro-Raman spectroscopy will give information on mineralogical composition of single particles. Micro-morphological and qualitative chemical analysis will be obtained through scanning electron microscopy/energy dispersive X-ray spectroscopy (SEM/ EDS) and transmission electron microscopy (TEM) on fine PM particles. Qualitative chemical composition will be carried out directly on filters by EDXRF analysis using Bruker TRACER IV equipment.

\section{Sample pre-treatment of PM}

Some sample pre-treatments are described in the literature, ${ }^{36}$ but they can cause sample losses. A simple purification will be used for the PM samples: the filter from the air sampler will be cut into halves and each divided into small pieces and put in a test tube containing $100 \mathrm{~mL}$ dichloromethane (DCM). Then, it will be placed in an ultrasonic bath $\left(4^{\circ} \mathrm{C}\right)$ for two cycles of $22 \mathrm{~min}$, and the solution will be filtered through a membrane (polytetrafluoroethylene (PTFE) $0.45 \mu \mathrm{m}$, Albet). The sample will be reduced to a final volume of $2 \mathrm{~mL}$ under low pressure. Acetonitrile/DCM (3/1, v/v) solution will be used to dissolve the residue for chromatographic analyses.

\section{HPLC measurements of PAHs in PM}

Pollutant analysis will be made by HPLC (HP 1100), equipped with an online diode array detector and a fluorescence detector (Kontron SFM). A sample or standard $(20 \mu \mathrm{L})$ will be injected onto a reverse-phase $\mathrm{C}_{18}$ column (Grace Vydac 201TP5415; $4.6 \mathrm{~mm} \times 150$ $\mathrm{mm}$ ). A linear gradient of water and acetonitrile (ACN; $\mathrm{v} / \mathrm{v}$ ) will be used to elute the compounds at a flow rate of $1 \mathrm{~mL} / \mathrm{min}$ as follows: for $0-2 \mathrm{~min}$, hold $40 \%$ ACN; for 2-40 min, a linear gradient from $40 \%$ to $100 \%$ ACN; and for $40-47 \mathrm{~min}$, hold $100 \%$ ACN. Two separate chromatography runs will be used to resolve the changing excitation/emission wavelengths of the PAHs in the fluorescence detector. The first run will be monitored at 265/389 nm from 0 to 28 min (for P, B[a]AN, CHR and $\mathrm{B}[\mathrm{e}] \mathrm{P}$ ) and $280 / 410 \mathrm{~nm}$ from 28 to $52 \mathrm{~min}$ (for $\mathrm{B}$ [ghi] PER, B[k]FA, B[b]FA, dB[ah]AN and B[a]P). Then, the second run will be monitored at $280 / 435 \mathrm{~nm}$ from 0 to $30 \mathrm{~min}$ (for FA) and $305 / 500 \mathrm{~nm}$ from 30 to $52 \mathrm{~min}$ (for Ind $[\mathrm{cd}] \mathrm{P}){ }^{36}$

\section{HPLC measurements of nitro-PAHs in PM}

Analysis will be made by high performance liquid chromatography (HPLC) (HP 1100), equipped with an online diode array detector, an electrochemical detector (ESA 5100A) and a fluorescence detector (Kontron SFM). A sample or standard $(20 \mu \mathrm{L})$ will be injected onto a reverse-phase $\mathrm{C}_{18}$ column (Hypersil ODS; $120 \AA$,
$5 \mu \mathrm{m} ; 4.6 \mathrm{~mm} \times 100 \mathrm{~mm})$. The mobile phase will be a solution of $0.1 \mathrm{M}$ sodium chloroacetate $(\mathrm{pH}$ 3) with $50 \%$ ACN $(\mathrm{v} / \mathrm{v})$ at a flow rate of $1 \mathrm{~mL} / \mathrm{min}$. The electrochemical detector parameters will be set as follows: conditioning cell-1.0 V (ESA 5021), detector I-0.5 V, detector II- $0.6 \mathrm{~V}$ (ESA 5011), response time- $0.1 \mathrm{~s}$, and gain $-1 \times 1$. Two separate chromatography runs will be used to resolve the changing excitation/emission wavelengths of the nitro-PAHs in the fluorescence detector. The first run will be monitored at $290 / 365 \mathrm{~nm}$ from 0 to $15 \mathrm{~min}, 302 / 530 \mathrm{~nm}$ from 15 to $25 \mathrm{~min}, 273$ / $440 \mathrm{~nm}$ from 25 to $37 \mathrm{~min}$, and $300 / 475 \mathrm{~nm}$ from 37 to $80 \mathrm{~min}$. The second run will be monitored at 263/ $505 \mathrm{~nm}$ from 0 to $15 \mathrm{~min}$ and $360 / 430 \mathrm{~nm}$ from 15 to $80 \mathrm{~min}$. The results, reported as molar concentrations, will be calculated after correlating the integrated trace peaks with their respective standard curves. ${ }^{36}$

\section{LINE-1 methylation analysis}

A blood sample will be collected and a white blood cell (WBC) count will be performed on fresh blood using automated methods within 3 hours from the time of blood drawing. DNA will be extracted using the Illustra blood genomic Prep Mini Spin Kit (GE Healthcare) according to the manufacturer's protocol and stored at $-20^{\circ} \mathrm{C}$. The LINE-1 methylation level in lymphocytes will be measured by pyrosequencing-based methylation analysis in three CpG sites of the LINE-1 promoter (GeneBank accession no. x58075), after DNA bisulfite conversion. ${ }^{15}$ The degree of LINE-1 methylation will be reported for each locus as well as the average percentage of methylation of the three evaluated.

\section{Cellular models}

A549 alveolar epithelial cells and alveolar macrophages isolated from healthy and non-smoker subjects will be used. The different types of PM will be treated with a 'Gamble Solution modified' that mimics the chemical and physical composition of the interstitial fluid deep regions of the lung (http://www.ivhhn.org) and tested as follows: (1) separate exposure of macrophages and A549 cells in culture at varying levels of PM for times varying from 4 to 48 hours; (2) after exposure to PM, the culture medium conditioned by macrophages will be used to grow non-exposed A549 cells in order to study the influence of the mediators released by macrophages; (3) the cellular toxicity of the PM in the cells treated in (1) will be measured by the lactate dehydrogenase (LDH) level released from cells A549; (4) nonradioactive quantification of cell proliferation and cell viability of A549 cells exposed to PM, by means of WST-1 colorimetric assay and direct microscopic counts; (5) evaluation of the expression of biomarkers of oxidative stress as heme-oxygenase-1 (HO-1) and pro-fibrotic marker as transforming growth factor $\beta$ (TGF-B) on the same cells by quantitative real-time PCR; (6) evaluation by ELISA of the release of a biomarker of inflammation as interleukin 8 (IL-8) in the culture medium 
conditioned; (7) assessment of LINE-1 methylation levels by pyrosequencing.

\section{Statistical analysis}

The study is designed to look at several exposureoutcome relationships (among all of them, the principals are: adherence to the MD and exposure to air pollutants). Given the lack of reference data on the association of these multiple exposures with LINE-1 methylation status, we assessed the statistical power based on previous results, evaluating the association between adherence to the MD and LINE-1 hypomethylation. ${ }^{19}$

Thus, in order to obtain at least $80 \%$ power with $95 \%$ CI, a total of 550 women will be enrolled.

The sample size and the power analysis have been computed using SPSS Sample Power. All collected data will be imputed in electronic databases and analysed using specific software (SPSS vs 22). The relationships between LINE-1 methylation levels and exposure variables (PM exposure, MD adherence and folate intake) will be initially assessed using the two-tailed $\chi^{2}$ test for the statistical comparison of proportions and to estimate the strength of associations by calculating the crude ORs and the corresponding 95\% CIs. Continuous variables will be tested using Student's t-test and one-way analysis of variance (ANOVA). Correlations will be assessed by Spearman's rank correlations. Multivariate analyses will be performed by fitting linear regression models adjusted by age, smoking status, BMI, employment and job type. In addition, the models will be adjusted for WBC count to explain for possible differences in the proportion of leucocyte subtypes. The adjusted ORs with their respective $95 \%$ CIs will be reported. A value of $\mathrm{p}<0.05$ will be considered statistically significant. After assessing the independent effects of PM exposure, MD adherence and folate intake on LINE-1 methylation levels, we will evaluate the combined effect of these exposures through various types of regression models.

In particular, multivariable linear regression models will be used to evaluate LINE-1 methylation levels in relation to PM exposure, MD adherence and folate intake, adjusting for age, smoking status, BMI, employment and WBC count. A comparison of the results will be performed taking into account seasonal variations of air pollutants. Furthermore, appropriate measures of interaction between risk factors will be computed using both stratified analysis and statistical models.

\section{ETHICS AND DISSEMINATION}

Participation in the study will be voluntary. All women participants will be fully informed of the purpose and procedures of the study, and a signed written consent will be obtained. All the data collected will be treated confidentially and analysed in an aggregate and anonymous way. The study protocol has been approved by the ethics committees of the involved institution and performed according to the Declaration of Helsinki.
The findings of the project will enable us to provide essential information for timely and effective public health action, and to provide for the development, recommendation and/or the implementation of appropriate interventions for the promotion of healthy eating behaviours, offering protection against air pollution effects. Results will be disseminated through peerreviewed journals and presentations at local, national and international conferences.

The main aim is to improve knowledge about environmental exposures and how they affect health outcomes, providing evidence to create healthy environments with an evident impact on public health and disease prevention. The proposed approach for this project is characterised by its multidisciplinary profile through the integration of specific methods and tools for the nutritional and molecular epidemiology characterisation, mineralogical and chemical analyses and in vitro assays, in order to assess whether the MD and the intake of specific nutrients can explain a portion of the attributable risk to air pollutants in a population of women living in the Catania metropolitan area.

The strength of the present project is that it integrates different individual exposures to investigate the associated epigenetic effects. The project design incorporates environmental and nutritional exposures in order to evaluate the associated epigenetic signatures of an individual's cumulative exposure.

This study has some limitations. The relationship between dietary patterns and DNA methylation may be confounded by genetic factors, such as the genetic polymorphism C677T in the MTHFR gene that may result in global DNA hypomethylation through an interaction with folate status. ${ }^{39}$ However, results from a recent meta-analysis did not show any conceivable evidence supporting the hypothesis that MTHFR C677T polymorphism contributes to DNA hypomethylation. ${ }^{26}$ Secondly, the assessment of folate intake through an FFQ does not preclude measurement errors and may suffer from inaccuracies of volunteers' recall. However, the FFQ used in this study has been specifically developed for use among our population and previously validated against a 4-day weighted dietary record. ${ }^{32}$ Thirdly, the peculiar geological and environmental setting, due to the volcanic components of the atmosphere, could be a potential confounder of the study. Being unable to perform a geographical comparison between volcanic and usual urban PM exposure, we have planned a mineralogical-chemical characterisation of PM in order to determine the major sources contributing to the PM abundance. Finally, although LINE-1 methylation has been proposed as a biomarker of global genomic methylation level, ${ }^{40}$ recent reports have shown that the methylation status of an individual sequence is not widely correlated with the global methylation level, due to a different sensitivity to environmental exposures. ${ }^{41}$ Accordingly, the expected results of the present research could provide the basis for future investigations on 
additional LINE-1 sequences, as well as DNA methylation changes in specific genes involved in PM exposure and nutrient intake. The long-term objective is part of the translational research, in terms of moving from basic research achievements into further knowledge about disease causes, risk and protective factors, to develop prevention strategies against environmentally related diseases. Women represent an important target for health promotion for biological and socio-cultural reasons. The transgenerational nature of epigenetic events suggests the pathway of transmission of the associated risk from mother to child. ${ }^{42}$ However, in animal models several studies have demonstrated phenotypic changes and different methylation levels in offspring based on the mother's diet during pregnancy, ${ }^{43}$ although in human populations this effect is not yet clear. Furthermore, women are commonly responsible for healthy lifestyles for their family and therefore the promotion of women's health potentially affects all stages of life.

\section{Author affiliations}

${ }^{1}$ Department of Medical and Surgical Sciences and Advanced Technologies

"GF Ingrassia", University of Catania, Catania, Italy

${ }^{2}$ LaPoSS, Laboratory of Policies and Social Services, University of Catania, Catania, Italy

${ }^{3}$ Department of Biological, Geological and Environmental Sciences, University of Catania, Catania, Italy

${ }^{4}$ Department of Chemical Science, Section of Photochemistry and

Photobiology, University of Catania, Catania, Italy

${ }^{5}$ Research Centre for the analysis, the monitoring and methodology for environmental risk assessment (CRAM3RA), University of Catania, Catania, Italy

${ }^{6}$ Department of Clinical and Experimental Medicine, University of Catania, Catania, Italy

Contributors $A A$ is the principal investigator of the project. $A A, G B, P M, G D G$ and $\mathrm{NC}$ conceived and designed the study. $\mathrm{MB}, \mathrm{AQ}, \mathrm{AM}, \mathrm{AC}$ and $\mathrm{MI}$ contributed to the design of the protocol and experiments. All the authors participated in the discussion of the protocol and read, edited, and approved the final version of the manuscript.

Funding The project was approved and funded by the University of Catania (Finanziamento della Ricerca, FIR 2014)

Competing interests None declared.

Provenance and peer review Not commissioned; externally peer reviewed.

Data sharing statement Data from the study will be available on request.

Open Access This is an Open Access article distributed in accordance with the Creative Commons Attribution Non Commercial (CC BY-NC 4.0) license, which permits others to distribute, remix, adapt, build upon this work noncommercially, and license their derivative works on different terms, provided the original work is properly cited and the use is non-commercial. See: http:// creativecommons.org/licenses/by-nc/4.0/

\section{REFERENCES}

1. Couto $E$, Boffetta $P$, Lagiou $P$, et al. Mediterranean dietary pattern and cancer risk in the EPIC cohort. Br J Cancer 2011:104:1493-9.

2. Wild CP. The exposome: from concept to utility. Int $J$ Epidemiol 2012;41:24-32.

3. Wolffe AP, Guschin D. Review: chromatin structural features and targets that regulate transcription. J Struct Biol 2000;129:102-22.

4. Hammoud SS, Cairns BR, Jones DA. Epigenetic regulation of colon cancer and intestinal stem cells. Curr Opin Cell Biol 2013;25:177-83.
5. Burris $\mathrm{HH}$, Baccarelli AA. Environmental epigenetics: from novelty to scientific discipline. J App/ Toxicol 2014;34:113-16.

6. Weisenberger DJ, Campan M, Long TI, et al. Analysis of repetitive element DNA methylation by MethyLight. Nucleic Acids Res 2005;33:6823-36.

7. Wallace NA, Belancio VP, Deininger PL. L1 mobile element expression causes multiple types of toxicity. Gene 2008;419:75-81.

8. Price EM, Cotton AM, Peñaherrera MS, et al. Different measures of "genome-wide" DNA methylation exhibit unique properties in placental and somatic tissues. Epigenetics 2012;7:652-63.

9. $\mathrm{Li} \mathrm{TH}$, Schmid CW. Differential stress induction of individual Alu loci: implications for transcription and retrotransposition. Gene 2001;276:135-41.

10. Kazazian $\mathrm{HH}$ Jr. Mobile elements: drivers of genome evolution Science 2004;303:1626-32.

11. Baba $\mathrm{Y}$, Huttenhower $\mathrm{C}$, Nosho $\mathrm{K}$, et al. Epigenomic diversity of colorectal cancer indicated by LINE-1 methylation in a database of 869 tumors. Mol Cancer 2010;9:125.

12. Woo HD, Kim J. Global DNA hypomethylation in peripheral blood leukocytes as a biomarker for cancer risk: a meta-analysis. PLoS One 2012;7:e34615.

13. Barchitta M, Quattrocchi A, Maugeri A, et al. LINE-1 hypomethylation in blood and tissue samples as an epigenetic marker for cancer risk: a systematic review and meta-analysis. PLoS One 2014;9:e109478.

14. Tamura T, Picciano MF. Folate and human reproduction. Am J Clin Nutr 2006;83:993-1016.

15. Piyathilake CJ, Badiga S, Kabagambe EK, et al. A dietary pattern associated with LINE-1 methylation alters the risk of developing cervical intraepithelial neoplasia. Cancer Prev Res (Phila) 2012;5:385-92. http://dx.doi.org/10.1158/1940-6207.CAPR-11-0387

16. Chou YF, Huang RFS. Mitochondrial DNA deletions of blood lymphocytes as genetic markers of low folate-related mitochondrial genotoxicity in peripheral tissues. Eur J Nutr 2009;48:429-36.

17. Wu MY, Kuo CS, Lin CY, et al. Lymphocytic mitochondrial DNA deletions, biochemical folate status and hepatocellular carcinoma susceptibility in a case-control study. Br J Nutr 2009;102:715-21.

18. Agodi A, Barchitta M, Quattrocchi A, et al. Folate deficiency is not associated with increased mitochondrial genomic instability: results from dietary intake and lymphocytic mtDNA 4977-bp deletion in healthy young women in Italy. Mutagenesis 2014;29:101-6.

19. Agodi A, Barchitta M, Quattrocchi A, et al. Low fruit consumption and folate deficiency are associated with LINE-1 hypomethylation in women of a cancer-free population. Genes Nutr 2015;10:480.

20. Piyathilake CJ, Macaluso M, Alvarez RD, et al. A higher degree of LINE-1 methylation in peripheral blood mononuclear cells, a one-carbon nutrient related epigenetic alteration, is associated with a lower risk of developing cervical intraepithelial neoplasia. Nutrition 2011;27:513-19.

21. Christensen BC, Kelsey KT, Zheng S, et al. Breast cancer DNA methylation profiles are associated with tumor size and alcohol and folate intake. PLoS Genet 2010;6:e1001043.

22. Kim JW, Park HM, Choi YK, et al. Polymorphisms in genes involved in folate metabolism and plasma DNA methylation in colorectal cancer patients. Oncol Rep 2010;25:167-72.

23. Wallace K, Grau MV, Levine AJ, et al. Association between folate levels and $\mathrm{CpG}$ island hypermethylation in normal colorectal mucosa. Cancer Prev Res 2010;3:1552-64.

24. Yamada K, Chen Z, Rozen R, et al. Effects of common polymorphisms on the properties of recombinant human methylenetetrahydrofolate reductase. Proc Natl Acad Sci USA 2001;98:14853-8.

25. Izmirli M. A literature review of MTHFR (C677T and A1298C polymorphisms) and cancer risk. Mol Biol Rep 2012;40:625-37.

26. Wang L, Shangguan S, Chang S, et al. Determining the association between methylenetetrahydrofolate reductase (MTHFR) gene polymorphisms and genomic DNA methylation level: a meta-analysis. Birth Defects Res Part A Clin Mol Teratol 2016;106:667-74

27. Baccarelli A, Wright RO, Bollati V, et al. Rapid DNA methylation changes after exposure to traffic particles. Am J Respir Crit Care Med 2009;179:572-8.

28. Byun HM, Panni T, Motta V, et al. Effects of airborne pollutants on mitochondrial DNA methylation. Part Fibre Toxicol 2013;10:18.

29. Pey J, Querol X, Alastuey A. Variations of levels and composition of PM10 and PM2.5 at an insular site in the Western Mediterranean. Atmospheric Res 2009;94:285-99.

30. Perez N, Pey J, Querol X, et al. Partitioning of major and trace components in PM10-PM2.5-PM1 at an urban site in Southern Europe. Atmospheric Environ 2008;42:1677-91.

31. Agodi A, Barchitta M, Valenti G, et al. Increase in the prevalence of the MTHFR 677 TT polymorphism in women born since 1959: 
potential implications for folate requirements. Eur J Clin Nutr 2011;65:1302-8.

32. Agodi A, Barchitta M, Valenti G, et al. Folate dietary intake and blood biomarkers reveal high-risk groups in a Mediterranean population of healthy women of childbearing potential. Ann Nutr Metab 2013;63:179-85.

33. Barchitta $M$, Quattrocchi A, Adornetto $V$, et al. Tumor necrosis factor-alpha $-308 \mathrm{G}>\mathrm{A}$ polymorphism, adherence to Mediterranean diet, and risk of overweight/obesity in young women. Biomed Res Int 2014;2014:742620.

34. World Health Organization. Physical status: the use and interpretation of anthropometry. Report of a WHO Expert Committee. World Health Organ Tech Rep Ser 1995;854:1-452.

35. Institute of Medicine Dietary Reference Intakes. Food and nutrition board. Washington DC: National Academy Press, 2001.

36. De Guidi G, Librando V, Minniti Z, et al. The PAH and nitro-PAH concentration profiles in size-segregated urban particulate matter and soil in traffic-related sites in Catania, Italy. Polycyclic Aromatic Compounds 2012;32:439-56.

37. Librando V, Bracchitta G, De Guidi G, et al. Photodegradation of anthracene and benzo[a]anthracene in polar and apolar media: new pathways of photodegradation. Polycyclic Aromatic Compounds 2014;34:263-79.

38. Pósfai M, Molnár Á. Atmospheric aerosol particles: a mineralogical introduction. Eur Mineralogical Union Notes Mineralogy 2013;13:213-94.

39. Friso S, Choi SW, Girelli D. A common mutation in the 5,10-methylenetetrahydrofolate reductase gene affects genomic DNA methylation through an interaction with folate status. Proc Nat/ Acad Sci USA 2002;99:5606-11.

40. Yang AS, Estecio MR, Doshi K, et al. A simple method for estimating global DNA methylation using bisulfite PCR of repetitive DNA elements. Nucleic Acids Res 2004;32:e38.

41. Wolff EM, Byun HM, Han HF, et al. Hypomethylation of a LINE-1 promoter activates an alternate transcript of the MET oncogene in bladders with cancer. PLoS Genet 2010;6: e1000917.

42. Haggarty P. Nutrition and the epigenome. Prog Mol Biol Trans/ Sci 2011;108:427-46.

43. Waterland RA, Jirtle RL. Early nutrition, epigenetic changes at transposons and imprinted genes, and enhanced susceptibility to adult chronic diseases. Nutrition 2004;20:63-8. 